\title{
Intelligent Recognition Method of Insufficient Fluid Supply of Oil Well Based on Convolutional Neural Network
}

\author{
Yanfeng $\mathrm{He}^{1^{*}}$, Zhenlong Wang1, Bin Liu ${ }^{2}$, Xiang Wang1, Bingchao $\mathrm{Li}^{2}$ \\ ${ }^{1}$ School of Petroleum Engineering, Changzhou University, Changzhou, China \\ ${ }^{2}$ The Eighth Oil Production Plant, Petrochina Changqing Oilfield Branch, Xi'an, China \\ Email: ^heyanfeng@cczu.edu.cn
}

How to cite this paper: He, Y.F., Wang, Z.L., Liu, B., Wang, X. and Li, B.C. (2021) Intelligent Recognition Method of Insufficient Fluid Supply of Oil Well Based on Convolutional Neural Network. Open Journal of Yangtze Gas and Oil, 6, 116-128. https://doi.org/10.4236/ojogas.2021.63011

Received: June 28, 2021

Accepted: July 25, 2021

Published: July 28, 2021

Copyright $\odot 2021$ by author(s) and Scientific Research Publishing Inc. This work is licensed under the Creative Commons Attribution International License (CC BY 4.0).

http://creativecommons.org/licenses/by/4.0/ (c) (i) Open Access

\begin{abstract}
Traditional methods for judging the degree of insufficient fluid supply in oil wells have low efficiency and limited accuracy. To address this problem, a method for intelligently identifying the degree of insufficient fluid supply in oil wells based on convolutional neural networks is proposed in this paper. Firstly, 5000 indicator diagrams with insufficient liquid supply were collected from the oilfield site, and a sample set was established after preprocessing; then based on the AlexNet model, combined with the characteristics of the indicator diagram, a convolutional neural network model including 4 layers of convolutional layers, 3 layers of down-pooling layers and 2 layers of fully connected layers is established. The backpropagation, ReLu activation function and dropout regularization method are used to complete the training of the convolutional neural network; finally, the performance of the convolutional neural network under different iteration times and network structure is compared, and the super parameter optimization of the model is completed. It has laid a good foundation for realizing the self-adaptive and intelligent matching of oil well production parameters and formation fluid supply conditions. It has certain application prospects. The results show that the accuracy of training and verification of the method exceeds $98 \%$, which can meet the actual application requirements on site.
\end{abstract}

\section{Keywords}

Degree of Insufficient Fluid Supply in Oil Wells, Indicator Diagram, Convolutional Neural Network, Alexnet, Backpropagation Algorithm, ReLu Activation Function, Dropout Regularization

\section{Introduction}

At present, most of my country's oil fields are in the middle and late stages of 
exploitation, and the shortage of oil wells is becoming more and more serious [1]. Insufficient fluid supply in oil wells usually causes empty pumping and hydraulic shock conditions. It does not only increase the risk of damage to the pumping equipment but also causes a waste of electrical energy, which virtually increases the development cost of the oil field. Therefore, accurately judging the degree of insufficient fluid supply in oil wells is essential for improving the above-mentioned problems and the efficiency of oil well production.

The degree of insufficient fluid supply in oil wells can be intuitively reflected in the dynamometer image of the pumping well. The dynamometer is the relationship curve between displacement and load in a reciprocating motion of the rod pump in the oil well. When there is insufficient fluid supply in the oil well, the lower right corner of the indicator graph curve will appear missing, showing a "knife handle", and the higher the fluid supply shortage, the more serious the missing. On-site personnel, based on experience, can intuitively judge the degree of insufficient liquid supply reflected in the power diagram, but it is difficult to accurately quantify. Meanwhile, due to limited human energy, it is impossible to conduct a comprehensive analysis of the massive indicator diagrams which can be automatically collected in real-time. To realize automated quantitative analysis, scholars at home and abroad have carried out research and proposed a series of methods, including 1) The method of judging the degree of insufficient liquid supply by using the area of the indicator diagram [2]. The higher the degree of insufficient liquid supply, the greater the lack of indicator diagrams, and the smaller the corresponding indicator diagram area. However, this method is difficult to distinguish similar indicator diagrams under different working conditions; 2) Judging the degree of insufficient liquid supply using effective stroke [3]. The ratio of the long side to the short side of the indicator diagram is the effective stroke, but the judgment of the long and short sides is more complicated, and it is difficult to automatically and accurately calculate the complex indicator diagram. In recent years, with the rapid development of a new generation of artificial intelligence technology, the example of convolutional neural networks in deep learning algorithms has attracted more and more attention from researchers. Compared with the traditional shallow neural network, the convolutional neural network can directly recognize pictures, avoid the influence of loss information caused by the extraction of feature values, and has achieved a large number of successful applications in image recognition and other fields [4]. In the field of petroleum engineering [5] [6] [7], oil well condition diagnosis based on the convolutional neural network has also achieved remarkable results [8] [9] [10]. Based on the above analysis, it can be predicted that analyzing the degree of insufficient fluid supply by using a convolutional neural network in oil wells has great potential.

To conclude, this paper proposes an intelligent recognition method of oil well fluid supply shortage based on a convolutional neural network. Combining the principle of convolutional neural network algorithm and the characteristics of 
the indicator diagram [11], the corresponding convolutional neural network architecture is researched and designed, and the recognition model is constructed in combination with the Softmax classifier. The model is trained and optimized on this basis. Experiments show that the accuracy of the model is over 98\%, which provides effective methods and tool support for the accurate and efficient analysis of insufficient fluid supply in oil wells.

\section{Convolutional Neural Network}

\subsection{The Basic Principles of Convolutional Neural Networks}

The basic principle of the convolutional neural network [12] [13]: Input the recognized target image, convolution operation to obtain the feature image; pooling operation to achieve dimensionality reduction of the image; repeated operations for multiple convolution and pooling to fully extract image features; The classification of the target image is completed in the fully connected layer. The convolution operation is mainly operated on the original image of $X$ to obtain a featured image, using $H_{\mathrm{i}}$ to represent the feature image of the i layer of the convolutional neural network. $H_{\mathrm{i}}$ can be expressed by the following formula:

$$
H_{\mathrm{i}}=f\left(H_{\mathrm{i}-1} * W_{\mathrm{i}}+b_{\mathrm{i}}\right) .
$$

In the formula, $W_{\mathrm{i}}$ represents the weight vector of the i layer convolution kernel, the symbol of ${ }^{*}$ represents the convolution operation of the image or feature map of the layer $i-1, b_{1}$ represents the offset vector of the i layer convolution kernel, and $f(x)$ represents the activation function.

\subsection{AlexNet Network Model}

In 1998, Yann Lecun proposed the classic LeNet-5 network model [14], which recognizes handwritten digits, and the input image size is $32 \times 32$. The network structure is configured as follows: the first and second convolutional layers are connected to the pooling layer respectively, and the third convolutional layer is connected to the two fully connected layers. As the first-generation classic convolutional neural network model, there is room for improvement and optimization of its network structure, convolution kernel size, activation function, and pooling kernel size in the diagnosis of insufficient fluid supply in oil wells.

AlexNet was created by Krizhevsky [15] [16] and others and won the ImageNet competition in 2012, which improved double the recognition accuracy of ImageNet. The model consists of an 8-layer structure and has outstanding advantages in image classification. The network structure of AlexNet is shown in Figure 1.

The network structure is matched as follows: the first, second, and fifth convolutional layers are connected to the pooling layer, the third and fourth convolutional layers are continuously convolved, finally, and the three fully connected 


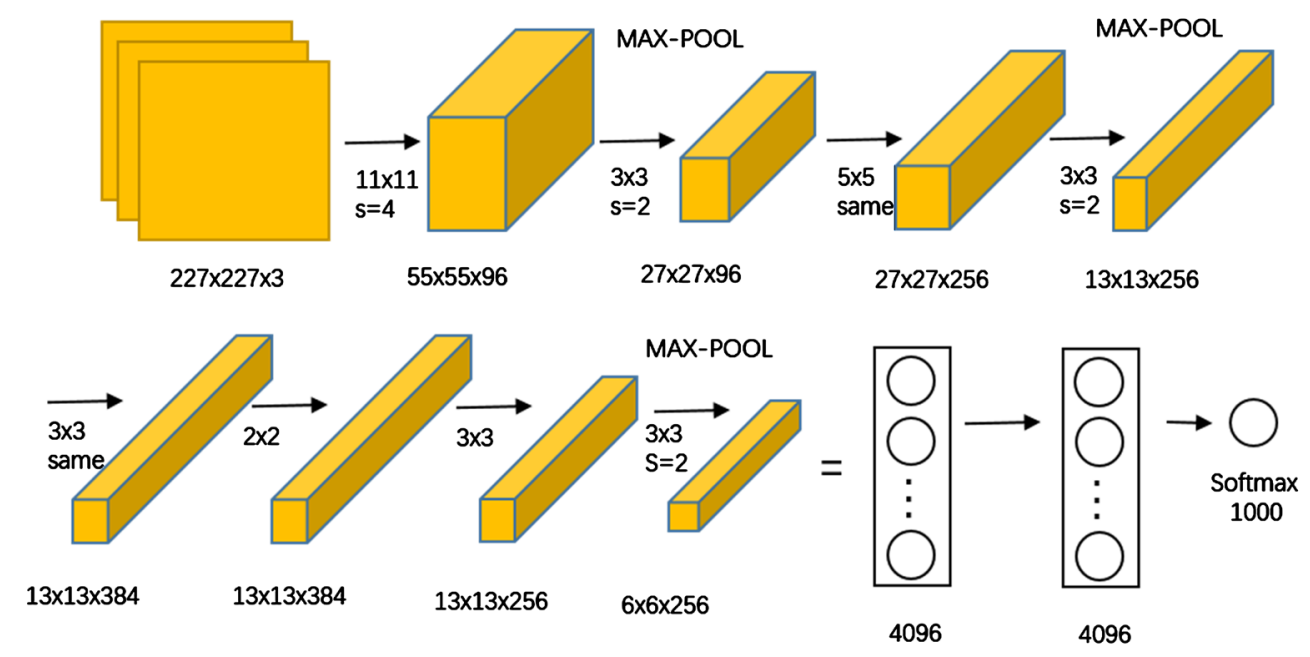

Figure 1. AlexNet neural network structure.

layers are connected. The AlexNet model is compared with the LeNet-5 model: A local response normalization mechanism (LRN) [17] is proposed. LRN creates a weight setting for the activity of local neurons in the model, which makes the image recognition process more effective Purposeful. The convolutional layer uses the ReLu activation function to replace the traditional Tanh activation function [18], which improves the accuracy and time of recognizing images. The pooling layer uses maximum pooling instead of traditional average pooling, which avoids the problem of blurring image feature extraction. The Dropout layer [19] is added to the fully connected layer to interrupt all connection parameters in proportion to prevent the occurrence of over-fitting. However, its relatively large network structure and large-size convolution kernel and pooling kernel still spend a lot of time in identifying the indicator diagram and the recognition accuracy is low.

\subsection{Improved AlexNet Network Model}

Aiming at the problem of diagnosing the degree of insufficient fluid supply in oil wells, the traditional Alexnet model has low recognition accuracy for similar indicator diagrams and takes more time. Therefore, by improving the AlexNet model [9] [20], it can not only meet the requirements of image recognition accuracy but also reduce the image recognition time. Based on the traditional AlexNet, there are mainly the following improvements:

1) The input size of the improved AlexNet model is $227 \times 227 \times 3$ pixels changed to $100 \times 200 \times 1$ pixels, and the 1000 neurons in the output layer are changed to 10 neurons;

2) The improved AlexNet model is composed of 4 layers of convolutional layers, 3 layers of pooling, and 2 layers of fully connected, which reduces training time and improves model convergence speed;

3) To improve the learning ability of the AlexNet model, a small convolution kernel with a size of $3 \times 3$ is used to replace the $5 \times 5$ and $11 \times 11$ convolution kernels in the original AlexNet network model, and a $2 \times 2$ pooling kernel is 
used Replace the $3 \times 3$ pooling core in the original AlexNet network model, increase the number of nonlinear operations, and enhance the ability of image feature learning. The improved AlexNet network structure is shown in Figure 2.

\section{Recognition of Insufficient Fluid Supply in Oil Wells Based on Convolutional Neural Network}

With the development of deep learning technology, convolutional neural networks perform well in the field of image recognition and are widely used. For judging the degree of insufficient fluid supply in oil wells, it is necessary to identify and classify the indicator diagrams. Therefore, the convolutional neural network is chosen as the diagnosis method.

\subsection{Insufficient Liquid Supply Indicator Diagram Pretreatment}

In the training process of the neural network, the indicator diagram needs to be preprocessed first. The quality of the preprocessing determines the level of the sample set and the training effect of the network. The specific steps are as follows:

1) Drawing and marking of the indicator diagram

This paper collects the suspension point load and displacement data of a rod pump in an oil field and draws it into a standardized indicator diagram: The size is $100 \times 200$ pixels, the DPI is 50 pixels/inches, the curve line width is 3 pixels, and the color black. Then classify and filter the indicator diagrams, and obtain 10 groups of indicator diagrams representing the degree of insufficient fluid supply in oil wells. As shown in Table 1.

2) Pixel normalization [21] [22]

The input original indicator diagram has pixels between 0 and 255, and the difference of the pixels will interfere with the learning ability of the neural network, creating an error invisibly. So further, this article uses the maximum and minimum normalization method, the formula is as follows.

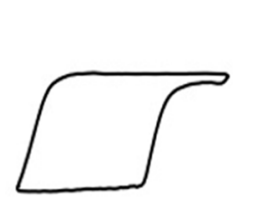

$100 \times 200 \times 1$

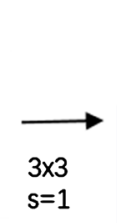

$98 \times 198 \times 16$

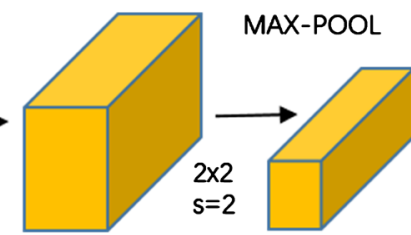

$49 \times 99 \times 16$

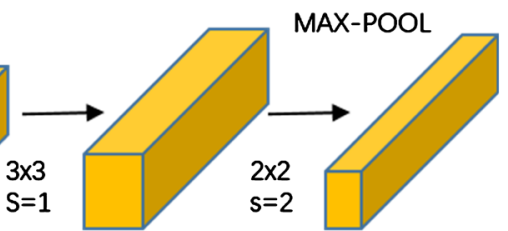

$47 \times 97 \times 32$

$23 \times 48 \times 32$

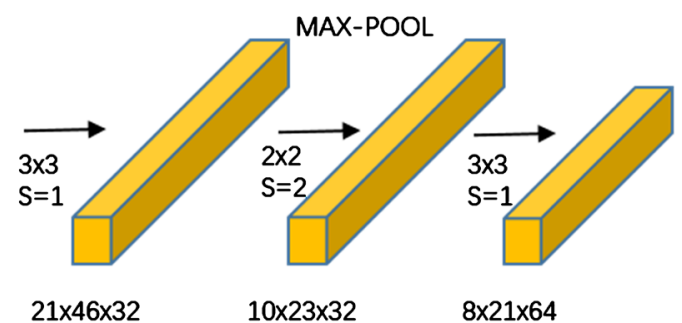

Figure 2. Improved AlexNet network model.

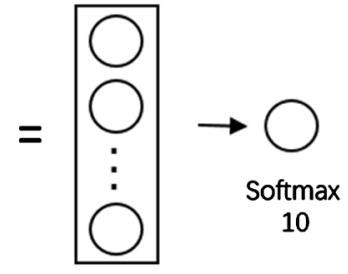

512 
Table 1. Degree of insufficient liquid supply.

\begin{tabular}{|c|c|c|c|c|}
\hline ID & Indicator diagrams & Liquid supply degree & Label & Data set/sheet \\
\hline 01 & & Normal & 10 & 500 \\
\hline 02 & & Level 9 & 9 & 500 \\
\hline 03 & & Level 8 & 8 & 500 \\
\hline 04 & & Level 7 & 7 & 500 \\
\hline 05 & & Level 6 & 6 & 500 \\
\hline 06 & & Level 5 & 5 & 500 \\
\hline 07 & & Level 4 & 4 & 500 \\
\hline 08 & & Level 3 & 3 & 500 \\
\hline 09 & & Level 2 & 2 & 500 \\
\hline 10 & & Level 1 & 1 & 500 \\
\hline
\end{tabular}

$$
\text { norm }=\frac{x_{\mathrm{i}}-\min (x)}{\max (x)-\min (x)}
$$

$x_{\mathrm{i}}$ is the image pixel value in the formula; $\min (x)$ and $\max (x)$ are the maximum and minimum values of image pixels.

The image is processed by the normalization method, and the pixels are returned to between 0 and 1 , without changing the image characteristics, and eliminating the difference between the wells. Make the required information more prominent, hide noise information, and save training time. 


\subsection{Recognition Process of Insufficient Liquid Supply Based on Convolutional Neural Network}

The flow chart for identifying the degree of insufficient fluid supply in oil wells designed in this paper is shown in Figure 3.

Step 1: First, the load and displacement data collected from the oil field production site are drawn into an indicator diagram. The indicator diagrams that do not meet the conditions are screened, and a sample set of indicator diagrams containing 10 types of insufficient liquid supply is established through the 5000 images obtained through the screening.

Step 2: Disrupt the sample set data, and divide the sample set into a training set and a validation set at a ratio of 8:2. According to the characteristics of the indicator diagram of insufficient liquid supply, a convolutional neural network structure is created.

Step 3: Input the training set and validation set data into the convolutional neural network model. Use the backpropagation algorithm to continuously update the weight parameters of the convolutional neural network to obtain the appropriate accuracy and recognition time, and save the trained model.

Step 4: Use the trained model to identify the indicator diagrams collected on site.

Step 5: For the wrong indicator diagram, correct the wrong recognition result. Put the corrected indicator diagram into the corresponding classification sample set, training the convolutional neural network again, and continuously optimizes the recognition model of the convolutional neural network.

\section{Experiments}

In this paper, a total of 5000 under-supply indicator diagrams are collected. First, the 5000 under-supply indicator diagrams are preprocessed according to the method described in section 2.1 of this article to obtain a sample set of 5000

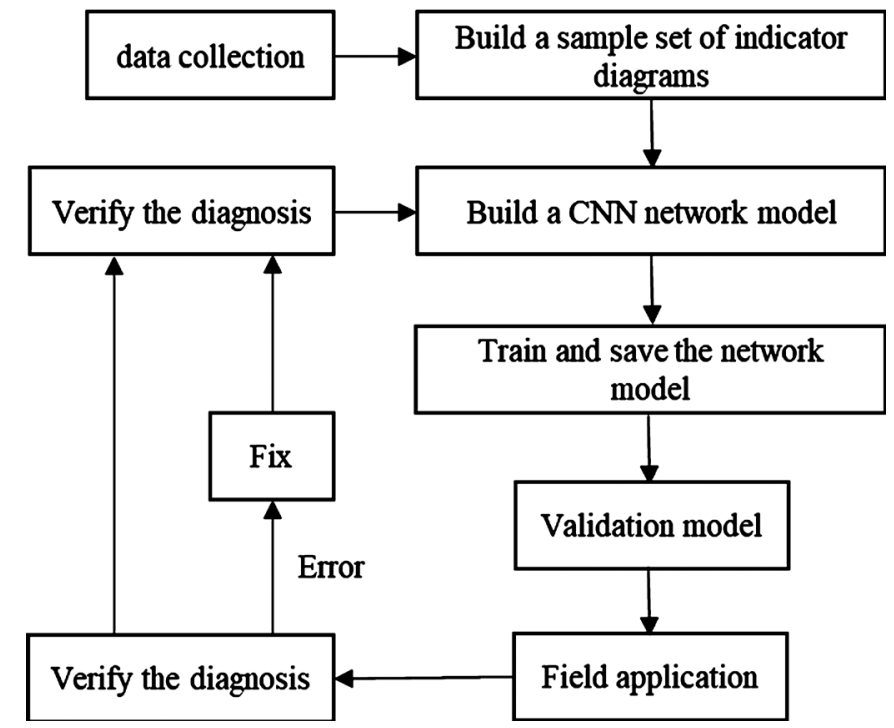

Figure 3. Identification process of insufficient fluid supply in oil wells. 
indicators with a size of $100 \times 200$. The experimental environment uses a 64-bit Win10 system; the computer's CPU is Intel(R)Core(TM)i7-10750K; the CPU's main frequency is $2.59 \mathrm{GHz}$; the memory is $16 \mathrm{~GB}$; the GPU is NVIDIA GeForce GTX 1650.

\subsection{Experimental Results}

In this experiment, the fast training method of multi-layer neural network based on GPU [23] [24], the training time needs $485 \mathrm{~s}$ in the case of GPU training, and $2501 \mathrm{~s}$ in the case of no GPU training. The number of iteration steps in the experiment is set to 200 steps, the learning rate is 0.0001 , and 200 images are learned each iteration. The total sample is divided into a training set and a verification set at a ratio of 8:2. The training set contains 4000 samples, and the verification set contains 1000 samples. The training accuracy and verification accuracy are shown in Figure 4 and Figure 5. We will standardize the confusion matrix by row before drawing, that is, the probability value is obtained, and the sum of all the probabilities in each row is 1 , so the diagonal line represents the recall rate (recall rate) of each category. The Confusion matrix is shown in Figure 6.

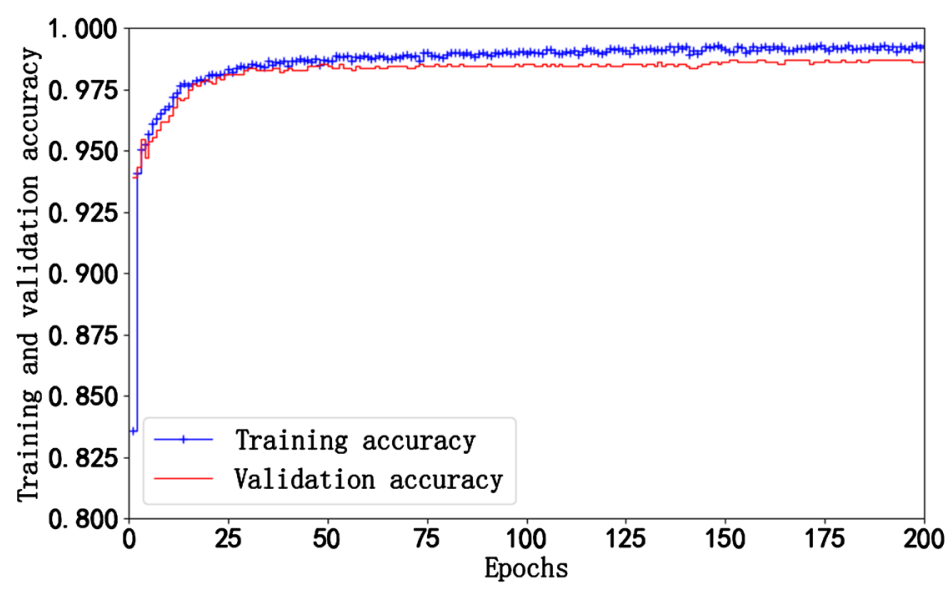

Figure 4. Training and verification accuracy.

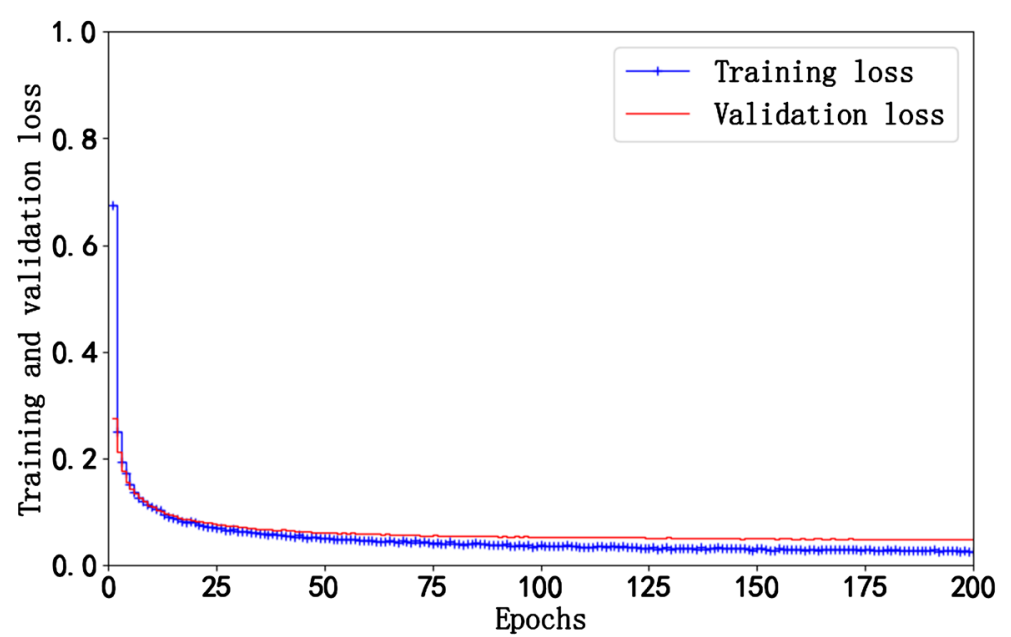

Figure 5. Training and verification loss results. 


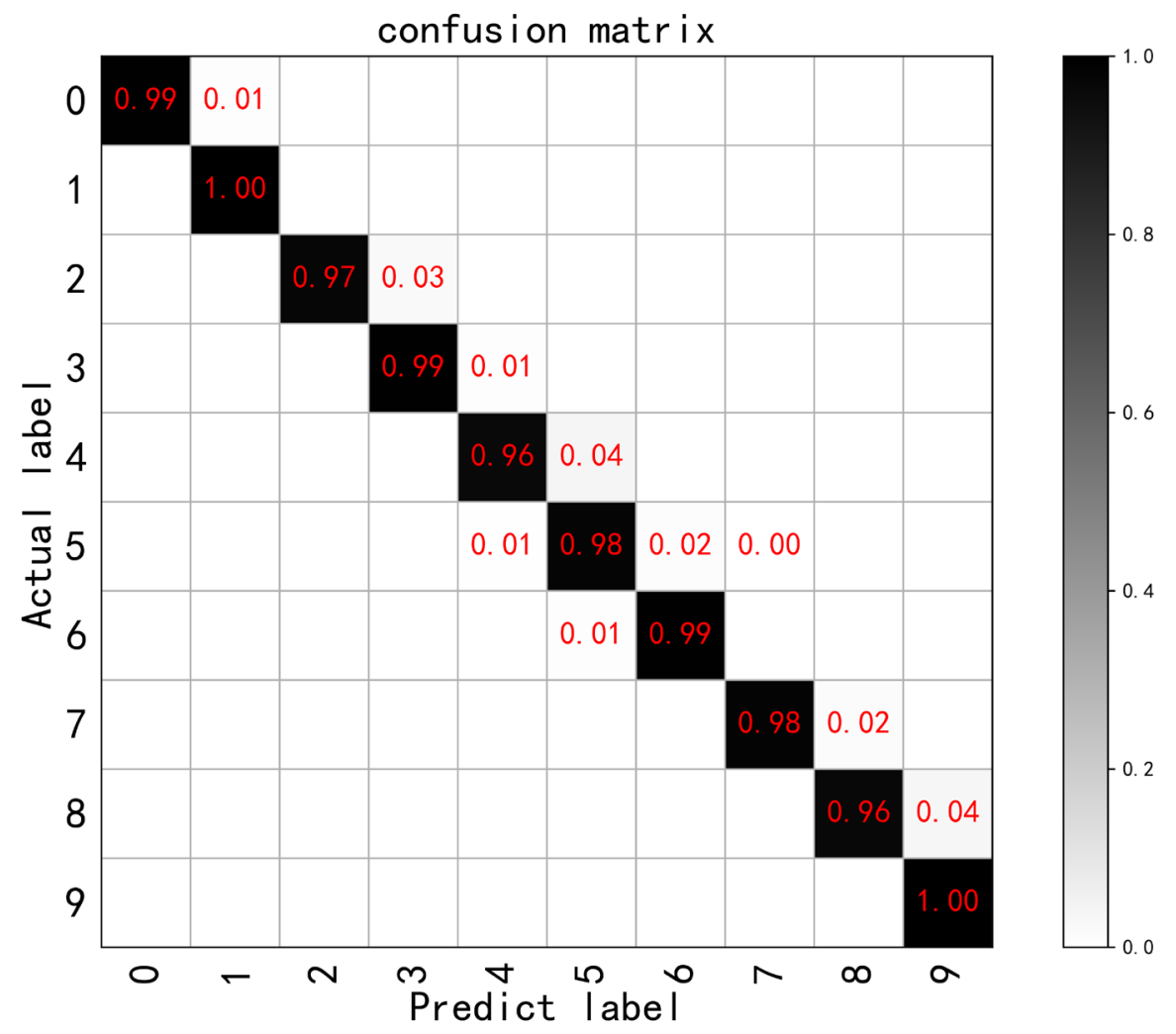

Figure 6. Confusion matrix.

\subsection{Analysis of Experimental Results}

The experiment was conducted with the sample set of the indicator diagram of insufficient liquid supply, and the learning was carried out 200 times. The network model converged about 75 times. It can be seen from Figure 4 that the initial training accuracy is on the rise, and after a period of time, the training accuracy remains above 99\%; from Figure 5, it can be seen that the initial training loss results have a downward trend. After a period of time, training the loss result is basically maintained at about 0.06 ; it can be seen that the training model is relatively successful. Using the validation set to verify the training model, it can be seen that after a period of time, the verification accuracy rate is basically maintained above $98 \%$, and the training loss result basically keeps it around 0.08 , which can meet actual functional requirements.

It can be seen from the confusion matrix that the recognition recall rate for 10 different degrees of insufficient liquid supply exceeds $90 \%$, which can meet the actual application requirements.

\subsection{Comparative Experiment}

In order to compare the performance of the convolutional neural network in this paper to identify the degree of insufficient liquid supply, the experiment verified the recognition performance of the other two methods on the same indicator diagram sample set, including the different iteration times and network structure of the convolutional neural network. 


\subsubsection{Comparison of Iteration Times}

As shown in Table 2, when the number of iterations is 100, compared with the number of iterations of 200 , the accuracy of the verification sample is increased by $1.51 \%$, which takes $165 \mathrm{~s}$ longer; when the number of iterations is 300 , compared with the number of iterations of 200, the accuracy of the verification sample is not much different. It takes 270s more time; when the number of iterations is 400 , compared with the number of iterations of 200 , there is little difference in the accuracy of the verification sample, which takes $313 \mathrm{~s}$ more time. Therefore, when the number of iterations is 200 , the model can be fully learned and converged.

Table 2. Comparison of different iteration times.

\begin{tabular}{ccc}
\hline Epochs & Validation accuracy & Time-consuming/s \\
\hline 100 & 0.9760 & 320 \\
200 & 0.9911 & 485 \\
300 & 0.9912 & 755 \\
400 & 0.9911 & 1068 \\
\hline
\end{tabular}

\subsubsection{Network Structure Comparison}

For convolutional neural networks, the structural ability is too strong, which will lead to data overfitting, because the number of layers increases. However, if the number of layers is too small and the structural capability is too weak, which will lead to underfitting of the data. In order to select the number of convolutional layers, the method of increasing layer by layer is used until the recognition rate no longer changes significantly as the number of layers increases, and the optimal number of layers can be determined. The first layer of this paper uses 16 convolution kernels with a size of $3 \times 3$, and the pooling layer uses $2 \times 2$ pooling kernels. The second layer uses 32 convolution kernels with a size specification of $3 \times 3$. The third layer uses 32 convolution kernels with a size specification of $3 \times$

3. The fourth layer uses 64 convolution kernels with a size specification of $3 \times 3$. The fifth layer uses 64 convolution kernels with a size specification of $3 \times 3$. As shown in Table 3.

The experimental results are shown in Table 4. As the number of convolutional layers increases, the recognition rate gradually increases, and then remains unchanged or decreases. Therefore, $\mathrm{C}$ is the most appropriate choice for the convolutional neural network structure, and the recognition rate is also the highest.

\section{Conclusions}

1) This paper, based on convolutional neural networks, proposes an intelligent analysis method for the degree of insufficient fluid supply in oil wells. Based on the indicator diagram data collected on the oilfield, a sample set of 10 indicator diagrams is established for the degree of insufficient fluid supply in oil wells. 
Table 3. Comparison of different network structures.

\begin{tabular}{|c|c|c|c|}
\hline \multicolumn{4}{|c|}{ ConvNet Configuration } \\
\hline A & B & $\mathrm{C}$ & $\mathrm{D}$ \\
\hline 2 weight layers & 3 weight layers & 4 weight layers & 5 weight layers \\
\hline \multicolumn{4}{|c|}{ Input $(100 \times 200 \times 1$ image $)$} \\
\hline Conv3-16 & Conv3-16 & Conv3-16 & Conv3-16 \\
\hline \multicolumn{4}{|c|}{ MaxPool } \\
\hline Conu2323 & Conv3-32 & Conv3-32 & Conv3-32 \\
\hline Convs-32 & Conv3-32 & Conv3-32 & Conv3-32 \\
\hline \multicolumn{4}{|c|}{ MaxPool } \\
\hline & & Conv3-64 & Conv3-64 \\
\hline \multicolumn{4}{|c|}{ MaxPool } \\
\hline & & & Conv3-64 \\
\hline \multicolumn{4}{|c|}{ FC-512 } \\
\hline \multicolumn{4}{|c|}{ Softmax-10 } \\
\hline
\end{tabular}

Table 4. Comparison of accuracy rates of different network structures.

\begin{tabular}{rr}
\hline Network structure & Validation accuracy \\
\hline A & 0.9421 \\
B & 0.9823 \\
C & 0.9911 \\
D & 0.9848 \\
\hline
\end{tabular}

2) In order to meet the needs of insufficient liquid supply indicator diagram recognition, the structure of the traditional AlexNet model is improved. The research results show that the recognition rate of the improved AlexNet model can reach $99.11 \%$, which improves the stability and accuracy of traditional methods for identifying insufficient fluid supply in oil wells, and greatly promotes safe production, environmentally friendly production, and efficient production in oil fields. In addition, the performance of the AlexNet model obtained from the experiment is closely related to the number of iterations and the network structure.

3) Training, verifying and optimizing the established neural network based on the sample set of "Indicator Diagram-Formation Fluid Supply Capability", an accurate and efficient intelligent quantitative analysis model of oil well fluid supply capability based on indicator diagram is formed. The self-adaptive and intelligent matching of oil well production parameters and formation fluid supply status has made a good foundation. It has certain application prospects.

\section{Fund Project}

Natural Science Research Project of Higher Education Institutions in Jiangsu Province (17KJB440001). 


\section{Conflicts of Interest}

The authors declare no conflicts of interest regarding the publication of this paper.

\section{References}

[1] Li, X., Fu, Z. and Liu, X.D. (2019) Research on the Reasonable Working System of Pumping Wells with Insufficient Liquid Supply. Petrochemical Technology, 26, $197+199$.

[2] Xu, X.Q., Zhou, H.B. and Li, M. (2017) Optimization and Adjustment Method of Pumping Unit Stroke Rate Based on the Area Change of the Hanging Point Indicator. Petrochemical Industry Automation, 53, 44-46.

[3] Qu, B.L. and Ma, W.G. (2018) Calculation Method and Influence Analysis of Fullness of Rod Pump. Petroleum Machinery, 46, 79-84.

[4] Li, X.Y., Xie, H.Y., Han, Z.H., Guo, H. and Fan, H. (2020) Research on Application of Convolutional Neural Network in Image Recognition Technology. Energy and Environmental Protection, 42, 73-76.

[5] Jiao, H.H. (2020) Research on Centrifugal Pump Fault Diagnosis Method Based on Hybrid Domain Features and Convolutional Neural Network. Thesis, Beijing University of Chemical Technology, Beijing.

[6] Du, Y. (2020) Research on Motor Bearing Fault Diagnosis Based on Deep Learning. Northeast Petroleum University, Heilongjiang.

[7] Tang, Z.G. (2019) Research on Fault Indicator Diagram Diagnosis Method Based on Convolutional Neural Network. Thesis, Xi'an University of Science and Technology, Xi'an.

[8] Wang, Y. (2020) Research on Intelligent Monitoring and Risk Prevention and Control Technology of Oil Wells. Henan Science, 38, 63-68.

[9] He, Y.F., Liu, C. and Wang, X. (2020) Application of Improved AlexNet Model in Fault Diagnosis of Rod Pump. Industrial Safety and Environmental Protection, 46, $22-26$.

[10] Wang, X., He, Y., Li, F., et al. (2021) A Working Condition Diagnosis Model of Sucker Rod Pumping Wells Based on Deep Learning. SPE Production \& Operations, 32, 1-10.

[11] Li, M.J., Li, Z., Qiu, L. and Qiu, Q. (2015) Application of Indicator Diagram Technology in Changqing Oilfield. Petrochemical Industry Automation, 51, 43-45.

[12] Lee, H. and Whang, M. (2018) Heart Rate Estimated from Body Movements at Six Degrees of Freedom by Convolutional Neural Networks. Sensors, 18, Article No. 1392. https://doi.org/10.3390/s18051392

[13] Ghosh, A., Sufian, A., Sultana, F., et al. (2020) Fundamental Concepts of Convolutional Neural Network. In: Balas, V., Kumar, R. and Srivastava, R., Eds., Recent Trends and Advances in Artificial Intelligence and Internet of Things, Springer, Cham, 519-567. https://doi.org/10.1007/978-3-030-32644-9 36

[14] El-Sawy, A., El-Bakry, H. and Loey, M. (2016) CNN for Handwritten Arabic Digits Recognition Based on Lenet-5. In: Hassanien, A., Shaalan, K., Gaber, T., Azar, A., Tolba, M., Eds., Proceedings of the International Conference on Advanced Intelligent Systems and Informatics, Springer, Switzerland, 566-575. https://doi.org/10.1007/978-3-319-48308-5 54

[15] Krizhevsky, A., Sutskever, I. and Hinton, G.E. (2012) ImageNet Classification with 
Deep Convolutional Neural Networks. Proceedings of the 25th International Conference on Neural Information Processing Systems, stand university, 2018, 1097-1105.

[16] Dhar, P., Dutta, S. and Vivekananda, M. (2021) Cross-Wavelet Assisted Convolution Neural Network (AlexNet) Approach for Phonocardiogram Signals Classification. Biomedical Signal Processing and Control, 63, Article ID: 102142. https://doi.org/10.1016/j.bspc.2020.102142

[17] Yuan, Y. (2017) Research on Image Quality Evaluation Method Based on Deep Convolutional Network. Thesis, Wuhan University, Wuhan.

[18] Wang, S.Y. and Teng, G.W. (2018) Optimization Design of ReLU Activation Function in Convolutional Neural Network. Information and Communication, 42-43.

[19] Zhang, W.F. and Zhou, J. (2019) Research on Fault Diagnosis of Rolling Bearing Based on Dropout-CNN. Light Industry Machinery, 37, 62-67.

[20] Zhao, X.Q. and Zhang, Q.Q. (2020) Improved Alexnet's Fault Diagnosis Method for Rolling Bearing under Variable Conditions. Vibration, Test and Diagnosis, 40, $472-480+623$.

[21] Gao, H.Y. (2018) Research and Application of Image Recognition Based on Machine Learning. Master's Thesis, Central China Normal University, Wuhan.

[22] Chen, T., Cheng, Y., Gan, Z., et al. (2021) Adversarial Feature Augmentation and Normalization for Visual Recognition.

https://www.researchgate.net/publication/350341593 Adversarial Feature Augmen tation and Normalization for Visual Recognition

[23] Bi, Z.J. (2015) Research on Parallel Accelerated Training Algorithm of Multi-GPU Multi-layer Neural Network. Thesis, Harbin Institute of Technology, Harbin.

[24] Wang, Y.M. (2016) Convolutional Neural Network Parallel Algorithm in Multi-GPU Environment. Thesis, University of Science and Technology of China, Hefei. 